\title{
Relevance of glueball bound states in the Yang-Mills plasma within a many-body $T$-matrix approach
}

\author{
Daniel Cabrera*t \\ Departamento de Física Teórica II, Universidad Complutense, 28040 Madrid \\ E-mail: daniel.cabrera@fis.ucm.es
}

\section{Gwendolyn Lacroix}

Service de Physique Nucléaire et Subnucléaire, Université de Mons - UMONS, Place du Parc 20, 7000 Mons, Belgium

E-mail: gwendolyn.lacroix@umons.ac.be

\section{Claude Semay}

Service de Physique Nucléaire et Subnucléaire, Université de Mons - UMONS, Place du Parc 20, 7000 Mons, Belgium

E-mail: claude.semay@umons.ac.be

\section{Fabien Buisseret}

Service de Physique Nucléaire et Subnucléaire, Université de Mons - UMONS, Place du Parc 20, 7000 Mons, Belgium;

Haute Ecole Louvain en Hainaut (HELHa), Chaussée de Binche 159, 7000 Mons, Belgium

E-mail: fabien.buissereteumons.ac.be

The strongly coupled phase of Yang-Mills plasma with gauge group $S U(3)$ is studied in a $T$ matrix approach. The existence of lowest-lying glueballs, interpreted as bound states of two transverse gluons (quasi-particles in a many-body setup), is analyzed in a non-perturbative scattering formalism with the input of lattice-QCD static potentials. The relevance of the singlet and the (colored) octet and $\mathbf{2 7}$ channels at finite temperature is discussed. We compute the equation of state of the system in Dashen, Ma and Bernstein's formulation of statistical mechanics and compare to quenched $S U(3)$ lattice data. Further analysis for the general case of $S U(N)$ is envisaged.

Sixth International Conference on Quarks and Nuclear Physics,

April 16-20, 2012

Ecole Polytechnique, Palaiseau, Paris

\footnotetext{
* Speaker.

${ }^{\dagger}$ The author acknowledges kind support from Service de Physique Nucléaire et Subnucléaire, Université de Mons, where this work was partly done.
} 


\section{Introduction}

The aim of the present work is to study the effect of two-body interactions in the thermodynamic features of the Yang-Mills (or gluon) plasma. We take recourse of Dashen, Ma and Bernstein's formulation of statistical mechanics [1] in terms of the $S$ - (or $T$-) matrix operator of gluon-gluon scattering. Correlations (leading to glueball bound states in the plasma) are studied within a non-perturbative approach which allows to investigate the behavior of the system in a range of temperatures where it is strongly interacting. We start by describing our setup for gluongluon interaction at finite temperature, and then apply our formalism to calculate the equation of state of the system. Next we discuss our result for the glueball spectrum in different color channels with special emphasis in dissociation temperatures and correlations above threshold. Finally, we compare our calculation of the pressure with recent lattice data in the pure gauge sector and discuss further applications of the present approach.

\section{Two (quasi-)gluon scattering in a $T$-matrix approach}

We follow Jacob and Wick's helicity formalism [2] in order to describe a two-gluon state, the gluons being considered as transverse spin-1 bosons. As shown in [3], two-gluon states can be organized in four families of helicity states in a $J^{P}$ basis, separated in helicity singlets $\left|S_{ \pm} ; J^{P}\right\rangle$ and doublets $\left|D_{ \pm} ; J^{P}\right\rangle$ (symmetrization for identical two-particle states imposes selection rules on $J$ ). Obviously, one cannot include all the possible $J^{P}$ channels contributing to the dynamics of the system. A valid criterion to select the most relevant ones is to keep low on the averaged orbital angular momentum. This has been used to interpret the mass hierarchy of the glueball spectrum [3] (in a simple nonrelativistic picture, it provides the strength of the centrifugal barrier in scattering theory). With $\left\langle\vec{L}^{2}\right\rangle=2$ one has, in terms of the standard $\left|{ }^{2 S+1} L_{J}\right\rangle$ basis,

$$
\left|S_{+} ; 0^{+}\right\rangle=\left[\frac{2}{3}\right]^{1 / 2}\left|{ }^{1} S_{0}\right\rangle+\left[\frac{1}{3}\right]^{1 / 2}\left|{ }^{5} D_{0}\right\rangle, \quad\left|S_{-} ; 0^{-}\right\rangle=-\left|{ }^{3} P_{0}\right\rangle,
$$

which in the singlet channel correspond to the $0^{++}$and $0^{-+}$glueballs, respectively. With $\left\langle\vec{L}^{2}\right\rangle=$ 4 one has the symmetric state $\left|D_{+} ; 2^{+}\right\rangle$plus three antisymmetric states: $\left|S_{+} ; 1^{-}\right\rangle,\left|S_{-} ; 1^{+}\right\rangle$and $\left|D_{-} ; 2^{-}\right\rangle$. The symmetric $2^{+}$state corresponds to the singlet $2^{++}$glueball, which is also among the lightest ones in vacuum. We shall keep no further states in our study.

The dynamics of gluon-gluon scattering in the plasma is encoded by the in-medium two-body scattering amplitude or $T$-matrix, which satisfies the (3D-reduced) Bethe-Salpeter equation (in partial waves)

$$
T_{J^{P}}\left(E ; q, q^{\prime}\right)=V_{J^{P}}\left(q, q^{\prime}\right)+\frac{1}{(2 \pi)^{3}} \int_{0}^{\infty} d k k^{2} V_{J^{P}}(q, k) G(E ; k) T_{J^{P}}\left(E ; k, q^{\prime}\right),
$$

with $q\left(q^{\prime}\right)$ the in-coming (out-going) momentum of a gluon in the center of mass reference frame and $E$ the total energy of the gluon pair. The two-body interaction potential in momentum space, $V_{J^{P}}$, is related to the potential in coordinate space, $V(r)$, by Fourier transform and partial wave projection. The resolvent function in Eq. (2.2) is given by the two-gluon propagator,

$$
G(E ; k)=\frac{m_{g}^{2}}{\varepsilon(k)} \frac{1}{\varepsilon(k)^{2}-E^{2} / 4+2 i \varepsilon(k) \Sigma}
$$


with the gluon dispersion relation $\varepsilon(k)=\sqrt{k^{2}+m_{g}^{2}}$ and $\Sigma$ is related to the imaginary part (i.e., decay width) of the single-particle gluon selfenergy in the plasma.

\section{Equation of state of the Yang-Mills plasma}

The $T$-matrix approach is particularly suited for a straightforward implementation of the contribution of two-body interactions in the partition function of the system. At vanishing chemical potential, the grand potential $\Omega$ of an interacting particle gas is given by [1]

$$
\Omega=\Omega_{0}+\sum_{v}\left[\Omega_{v}-\left.\frac{1}{2 \pi^{2} \beta^{2}} \int_{M_{v}}^{\infty} \frac{d \varepsilon}{4 \pi i} \varepsilon^{2} K_{2}(\beta \varepsilon) \operatorname{Tr}_{v}\left(\mathscr{S} S^{-1} \overleftrightarrow{\partial_{\varepsilon}} S\right)\right|_{c}\right]
$$

The first term, $\Omega_{0}$, is the grand potential of a gas of free relativistic particles (i.e., when the interactions are turned off). The second term accounts for interactions in the plasma. The sum runs on all the quantum numbers $(v)$ necessary to fix a scattering channel. Below threshold, the contribution from bound states leads to the $\Omega_{v}$ term, behaving as free additional species in the plasma. The third term accounts for the scattering contribution above threshold. The operator $\mathscr{S}$ enforces symmetrization in those channels involving identical particles, and the subscript $c$ means that only connected scattering contributions are taken into account. The scattering operator is defined as $S=1-2 \pi i \delta\left(\varepsilon-H_{0}\right) T$, with $H_{0}$ is the free Hamiltonian of the system. It is worth mentioning that the scattering contribution sets in at the level of the second order coefficient in a virial expansion of the partition function. Also note that Eq. (3.1) can be rewritten, by means of unitarity of the $S$-matrix, in terms of a weighted thermal average of scattering phase shifts. Once the off-shell $T$-matrix is known, the pressure is simply given by $p=-\Omega$. Other thermodynamic observables can be derived form $p$. For example, the trace anomaly $\Delta=e-3 p$ and the entropy density $s$ read, respectively, $\Delta=-\frac{1}{\beta^{3}}\left[\partial_{\beta}\left(\beta^{4} p\right)\right]_{V, \beta \mu}$ and $s=-\beta^{2}\left[\partial_{\beta} p\right]_{V, \mu}$.
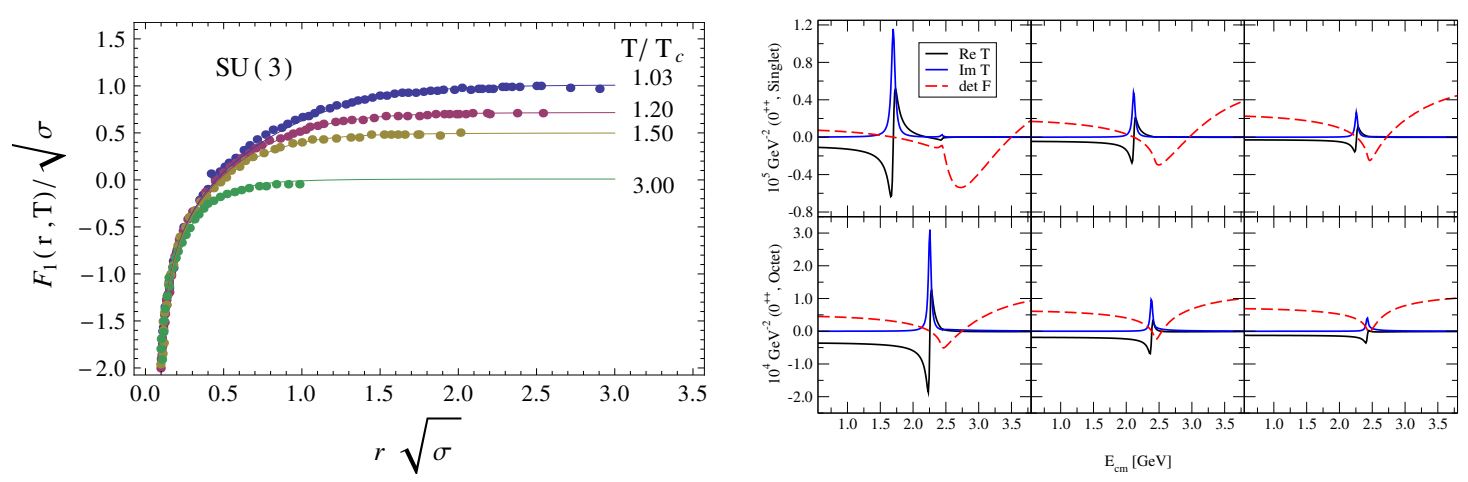

Figure 1: Left: Static free energy $F_{1}(r, T)$ of a quark-antiquark pair bound in a color singlet, computed in SU(3) quenched lattice QCD [4]. Solid lines are given by an appropriate parameterization [5]. Right: $T$-matrix for $g g$ scattering in the $0^{++}$singlet and octet channels. From left to right the temperatures are $(1.05,1.10,1.15) T_{c}$.

\section{Glueballs above $T_{c}$}

Following the idea in [6] we take recourse of lattice QCD simulations to have an estimate of the finite-temperature gluon-gluon interaction potential from first principles. Accurate computations 
of the static free $\left(F_{1}\right)$ and internal $\left(U_{1}\right)$ energies of a massive quark-antiquark pair in a color singlet state are available [4], particularly in quenched $S U(3)$ simulations, which come handy for our purpose (see Fig. 1, left panel). Whether it is $F_{1}$ or $U_{1}$ (or none) the most appropriate potential to be identified with the in-medium two-body interaction at finite temperature cannot be decided a priori from field-theoretical arguments. Still, as in [6], we choose $U_{1}$, which typically provides a more attractive interaction leading to larger dissociation temperatures (and thus results using this potential should be understood as an upper bound). We assume color scaling and derive the interaction potential between two quasi-gluons in color channel $\mathscr{C}$ as

$$
V(r, T)=\frac{\kappa_{\mathscr{C} ; g g}}{\kappa_{q \bar{q}}}\left[U_{1}(r, T)-U_{1}(\infty, T)\right]
$$

where $\kappa_{q \bar{q}}$ is the color factor of the singlet quark-antiquark pair, and $\kappa_{\mathscr{C} ; g g}=\left(C_{2}^{\mathscr{C}}-2 N\right) / 2 N$ for $S U(N)$, with $C_{2}^{\mathscr{C}}$ the quadratic Casimir of the gluon pair in representation $\mathscr{C}$. As is customary, the potential is normalized to zero as to ensure convergence of the scattering equation.

The only remaining unknown in our approach is the (effective) quasi-gluon mass. At finite temperature, it is well accepted that the infinite separation distance plateau in $U_{1}$ may be interpreted as an in-medium contribution (selfenergy) to the gluon mass [7]. The minimal implementation is a shift to the bare (zero temperature) gluon mass, which at the same time has to be fixed, for instance, by reproducing the glueball spectrum in vacuum. In terms of the asymptotic value of $U_{1}$, we propose the mass shift to be

$$
m_{g}(T)^{2}=\left(m_{g}^{0}\right)^{2}+\delta(T)^{2}, \quad \delta(T)=\sqrt{\frac{C_{g}}{C_{q}}} \frac{U_{1}(\infty, T)}{2} .
$$

We neglect at the moment the momentum dependence of this correction, which could be addressed in a full many-body study of the gluon dispersion relation. $\delta(T)$ scales with the one-body Casimir as suggested by the single-particle character of the correction (using two-body scaling, as in the potential, leads to a channel dependent mass correction). The structure of the color factor, $\sqrt{C_{g} / C_{q}}$, leads to the correct large- $N$ limit of the gluon mass (as it is the case for the Hard-Thermal-Loop result at high temperatures). Also note that this structure emerges if the gluon selfenergy is calculated from the $T$-matrix in a Brueckner scheme.

\begin{tabular}{c|rrr} 
State & Lattice [8] & $T$-matrix & CGQCD [9] \\
\hline $0^{++}$ & $1.73(5)(8)$ & 2.17 & 1.98 \\
$0^{-+}$ & $2.59(4)(13)$ & 2.39 & 2.22 \\
$2^{++}$ & $2.40(2.5)(12)$ & 2.34 & 2.42
\end{tabular}

Table 1: Masses (in GeV) of the lowest-lying glueball states at zero temperature. Our results (third column), are compared to the lattice data of Ref. [8] and to the Coulomb gauge QCD study in [9].

First of all, we test that our approach provides reasonable results in vacuum and, thus, is a solid starting point for the finite temperature study. We use the "funnel" potential form with string breaking setting in at $V_{s b} \simeq 2 \mathrm{GeV}$ as suggested by the value of (twice) the lightest gluelump mass. Our results for the ground states in $0^{++}, 0^{-+}$and $2^{++}$channels are shown in Table 1 , together with lattice results from [8] and the Coulomb-gauge QCD calculation in [9] (which formally shares 
many features with our $T$-matrix formulation). A few comments are in order: the parameters of the potential, $\alpha$ and $\sigma$, are optimized for the best fit of the lattice data for $F_{1}$ beyond $T_{c}$. This justifies the slightly too high value of the energy of the $0^{++}$glueball. A more precise agreement with the lattice glueball spectrum (including excited states) can be achieved if tuning the vacuum potential parameters independently of the finite temperature fit. More details are to be discussed in [5].

In Fig. 1 (right panel) we show the $T$-matrix, at several temperatures, for the singlet and octet scalar glueball channels. In this plot $m_{g}$ is set to $1.22 \mathrm{GeV}$ (namely, we use $m_{g}^{0} \simeq 0.7 \mathrm{GeV}$ and $\delta=$ $V_{s b} / 2$, as in vacuum), thus the medium effects stem only from the temperature dependence of the potential. The goal is to illustrate the mechanism of dissociation by color screening: as temperature is increased, the bound states progressively move towards the two-particle threshold and eventually dissolve by turning to scattering states. The red, dashed curve represents $\operatorname{det}(1-V G)$ and vanishes exactly at the bound state energy. It also signals resonant states beyond threshold.
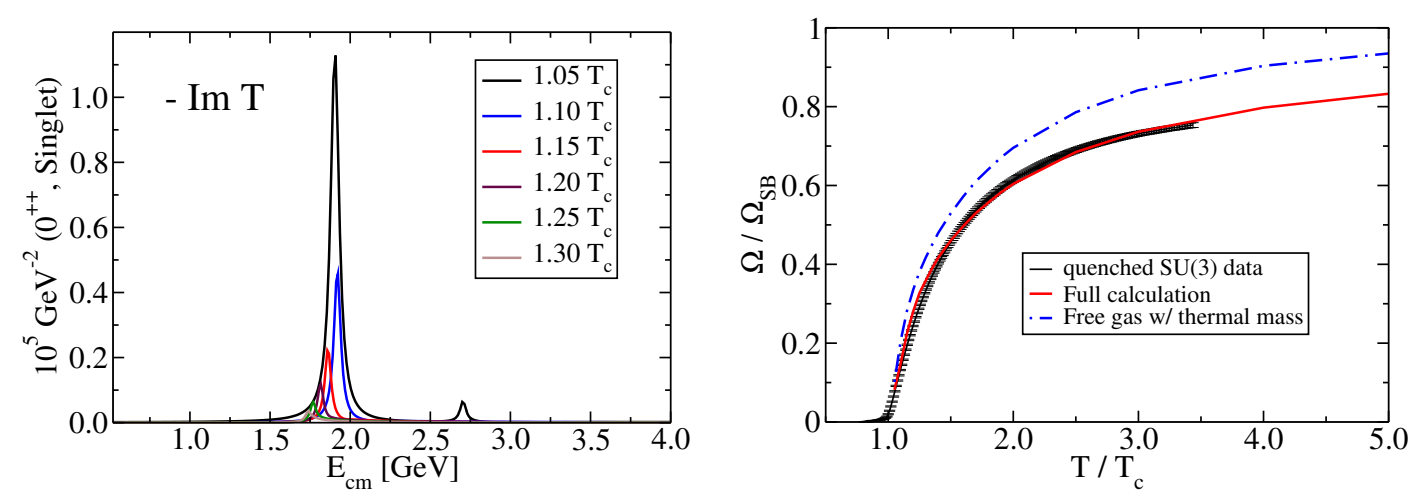

Figure 2: Left: $\operatorname{Im} T$ for $g g$ scattering in the $0^{++}$singlet channel with in-medium quasi-gluon masses (see text). Right: Pressure of the $S U(3)$ gluon-glueball gas. Lattice data from [11]. The dashed curve represents the pressure of the free quasi-gluon gas (no $g g$ interactions).

Next we turn the in-medium gluon mass correction on, $\delta(T)$. The bare gluon mass parameter is kept to $m_{g}^{0} \simeq 0.7 \mathrm{GeV}$. This is determined by our best comparison to the pure gauge lattice QCD data on the pressure, $p=-\Omega$ (see discussion below). The results for the imaginary part of the $T$-matrix in the singlet scalar channel are displayed in Fig. 2 (left panel). Two competing effects are responsible for the temperature evolution of the spectrum: reduction of the binding energy and downward shift of the threshold energy. Overall, the singlet scalar bound state experiences a mild shift to lower energies and dissociates at about $1.3 T_{c}$ (this is the value from which $\operatorname{det} F \operatorname{does}$ not vanish anymore; still, considerable strength remains at threshold up to about $1.5 T_{c}$ ). This is in qualitative agreement with the spectral function analysis of Euclidean correlators by the CLQCD Collaboration [10]. We also find bound states in the scalar octet channel, although considerably less bound. We find bound states in the pseudoscalar singlet and octet channels lying right below the threshold energy at the lowest considered temperature of $1.05 T_{c}$. The differences between singlet and octet channels are attributed to the strength of the potential, which scales with the twobody Casimir. In particular, note that the $\mathbf{2 7}$ channel is repulsive, and thus no bound states are found. Also note that states in the pseudoscalar channels, which in our approach correspond to pure $P$-wave scattering, are just mildly bound due to the centrifugal barrier. The tensor states, having an $S$-wave component, lie between the scalar and pseudoscalar channels regarding binding 
and dissociation temperatures. Finally, we compute the partition function of the gluon-glueball gas in the formalism of Dashen et al., as discussed above. The results, normalized to the StefanBoltzmann pressure, are shown in Fig. 2 (right panel). Note that, strictly speaking, the only free parameter in the calculation is the bare gluon mass, $m_{g}^{0}$. A value of $0.7 \mathrm{GeV}$ provides a remarkable agreement with the quenched $S U(3)$ lattice data from Panero [11]. In order to estimate the effect of two-body interactions, the partition function for a free gas including thermal mass corrections is also shown. Note that our bound states dissolve relatively quickly and only contribute significantly at temperatures at most up to $\simeq 1.5 T_{c}$. This illustrates the relevance of accounting for strong correlations from scattering in the continuum, a contribution which is readily addressed in our $T$-matrix formalism.

\section{Summary and outlook}

The relevance of gluon-gluon interactions beyond the critical temperature in the pure gauge $S U$ (3) plasma has been addressed in a non-perturbative $T$-matrix many-body framework with the input of (Casimir-scaled) potentials from thermal lattice QCD. We find glueball bound states in the singlet and octet channels surviving up to temperatures of about $1.3-1.5 T_{c}$, together with sizable threshold effects due to strong correlations beyond the two-particle threshold. With a minimal number of parameters, we reproduce the equation of state of the gluon-glueball gas in good agreement with recent quenched $S U(3)$ simulations.

The $T$-matrix formalism can be applied to calculate bulk thermodynamical properties of the system such as the sheer viscosity, which can be easily computed in relaxation-time approximation within a quasi-particle picture. Our approach also allows for a straightforward extension to $S U(N)$ analysis. A study of the exceptional group $G_{2}$, of theoretical interest related to the origin of (de)confinement, is also in progress.

\section{References}

[1] R. Dashen, S.-k. Ma and H. J. Bernstein, Phys. Rev. 187, 345 (1989).

[2] M. Jacob and G. C. Wick, Annals Phys. 7, 404 (1959).

[3] T. Barnes, Z. Phys. C 10, 275 (1981); V. Mathieu, F. Buisseret and C. Semay, Phys. Rev. D 77, 114022 (2008).

[4] O. Kaczmarek, F. Karsch, P. Petreczky and F. Zantow, Phys. Lett. B 543, 41 (2002).

[5] D. Cabrera, G. Lacroix, C. Semay, F. Buisseret, in preparation.

[6] D. Cabrera and R. Rapp, Phys. Rev. D 76 (2007) 114506; H. van Hees et al., Phys. Rev. Lett. 100 (2008) 192301; F. Riek and R. Rapp, Phys. Rev. C 82 (2010) 035201.

[7] A. Mocsy and P. Petreczky, Phys. Rev. D 73, 074007 (2006); S. Gupta, K. Huebner and O. Kaczmarek, Phys. Rev. D 77 (2008) 034503.

[8] C. J. Morningstar and M. J. Peardon, Phys. Rev. D 60 , 034509 (1999); Y. Chen et al., Phys. Rev. D 73, 014516 (2006).

[9] A. P. Szczepaniak and E. S. Swanson, Phys. Rev. D 65, 025012 (2002).

[10] X. -F. Meng et al. Phys. Rev. D 80 (2009) 114502.

[11] M. Panero, Phys. Rev. Lett. 103, 232001 (2009). 